OPEN ACCESS

Edited by:

Paulo Correia-de-Sá, University of Porto, Portugal

Reviewed by: Guillermo Diaz-Araya, University of Chile, Chile Guy Salama, University of Pittsburgh, United States

${ }^{*}$ Correspondence: Anita A. Pinar anita.pinar@monash.edu

Chrishan S. Samuel chrishan.samuel@monash.edu

Specialty section: This article was submitted to Cardiovascular and Smooth Muscle Pharmacology, a section of the journal Frontiers in Pharmacology

Received: 12 March 2020

Accepted: 23 July 2020 Published: 04 August 2020

Citation:

Pinar AA, Yuferov A, Gaspari TA and Samuel CS (2020) Relaxin Can Mediate Its Anti-Fibrotic Effects by Targeting the Myofibroblast NLRP3 Inflammasome at the Level of Caspase-1.

Front. Pharmacol. 11:1201. doi: 10.3389/fphar.2020.01201

\section{Relaxin Can Mediate Its Anti-Fibrotic Effects by Targeting the Myofibroblast NLRP3 Inflammasome at the Level of Caspase-1}

\author{
Anita A. Pinar ${ }^{1 *}$, Alexander Yuferov ${ }^{1}$, Tracey A. Gaspari ${ }^{1}$ and Chrishan S. Samuel ${ }^{1,2^{*}}$ \\ ${ }^{1}$ Cardiovascular Disease Program, Monash Biomedicine Discovery Institute and Department of Pharmacology, Monash \\ University, Clayton, VIC, Australia, ${ }^{2}$ Department of Biochemistry and Molecular Biology, The University of Melbourne, \\ Parkville, VIC, Australia
}

Introduction: The NLRP3 inflammasome produces interleukin (IL)-1 $\beta$ and IL-18, which when chronically activated by transforming growth factor (TGF)- $\beta 1$, contribute to fibrosis. The recombinant form of the anti-fibrotic hormone, relaxin $(R L X)$, suppresses the profibrotic influence of TGF- $\beta 1$ and toll-like receptor (TLR)-4 on NLRP3 inflammasome priming and activity in human cardiac myofibroblasts and mice with cardiomyopathy. However, whether RLX also modulates components of the myofibroblast NLRP3 inflammasome remains unknown.

Methods and Results: Stimulation of a human dermal fibroblast (HDF) cell line with TGF$\beta 1$ [5 ng/ml; to promote myofibroblast (HDMF) differentiation], LPS (100 ng/ml; to prime the NLRP3 inflammasome) and ATP ( $5 \mathrm{mM}$; to activate the NLPR3 inflammasome) ( $T+\mathrm{L}$ +A) significantly increased NLRP3 inflammasome priming and activity after 8 and 72 h; and $\alpha$-SMA expression (myofibroblast differentiation) and collagen-I deposition after $72 \mathrm{~h}$. siRNA-induced knock-down of NLRP3 inflammasome priming components (NLRP3, ASC, caspase-1) in T+L+A-stimulated HDMFs for $24 \mathrm{~h}$, completely knocked-down each component after $72 \mathrm{~h}$. RLX $(100 \mathrm{ng} / \mathrm{ml})$ administration to $T+L+A$-stimulated HDMFs after control, NLRP3 or ASC siRNA transfection, equivalently suppressed IL$1 \beta$, pro-IL-18, $\alpha-S M A$, and collagen-I protein levels (by $40 \%-50 \%$; all $p<0.05$ vs. $T+L+A$ ) after $72 \mathrm{~h}$, as determined by Western blotting. These RLX-induced effects were abrogated by siRNA knock-down of caspase-1.

Conclusion: The anti-fibrotic actions of RLX appear to require modulation of caspase- 1 within the myofibroblast NLRP3 inflammasome.

Keywords: fibrosis, myofibroblasts, NLRP3 inflammasome, caspase-1, relaxin 


\section{INTRODUCTION}

Inflammation is initiated by the innate immune system as part of a natural wound healing response to tissue injury. The infiltration of activated innate immune cells (Wynn, 2008) and their ability to secrete pro-inflammatory and profibrotic cytokines and growth factors such as transforming growth factor (TGF)- $\beta 1$ and several interleukins (ILs) including IL- $1 \beta$ and IL-18 are initiated to facilitate wound healing. However, when these factors are over-activated, they eventually contribute to fibrosis (Kolb et al., 2001; Gasse et al., 2007). These pro-fibrotic cytokines stimulate extracellular matrix (ECM)-producing fibroblasts to proliferate and differentiate into activated myofibroblasts (Wynn, 2008). These chronically activated myofibroblasts interact with the ECM and deposit large amounts of ECM proteins, predominantly collagens, to facilitate wound healing. However, the overproduction of ECM deposition contributes to anomalous tissue remodeling and scarring that displaces the integrity of the healthy tissue (Wynn and Ramalingam, 2012; Schroer and Merryman, 2015; Li et al., 2018; Frangogiannis, 2019; Humeres and Frangogiannis, 2019).

The nucleotide-binding oligomerization domain, leucinerich repeat and pyrin domain-containing protein 3 (NLRP3) inflammasome is a large multiprotein complex (Mangan et al., 2018; Zhou et al., 2018) that is expressed by several cell types including macrophages (Rodriguez-Alcazar et al., 2019), epithelial cells, microvascular endothelial cells and myofibroblasts (Boza et al., 2016). The NLRP3 inflammasome is formed to mediate the wound healing response to tissue injury via the production of IL-1 $\beta$ and IL-18. However, when chronically activated, it has emerged as a key contributor to fibrosis progression (Kolb et al., 2001). The NLRP3 inflammasome consists of the sensor molecule NLRP3, the adaptor protein apoptosis-associated speck-like protein containing a c-terminal caspase-recruitment domain (ASC), and the effector protease, caspase- 1 . The maturation and release of IL- $1 \beta$ is instigated via a two-step signaling process. The first "priming signal" synthesizes pro-IL-1 $\beta$ and pro-IL-18 and upregulates the expression of NLRP3 inflammasome components including NLRP3, ASC and pro-caspase- 1 . The second "activation signal" assembles the components of the NLRP3 inflammasome into the tri-partite protein complex, resulting in its activation, caspase- 1 activation, and subsequent maturation and secretion of IL-1 $\beta$ and IL-18. TGF- $\beta 1$ and toll-like receptor (TLR)- 4 are known inducers of the NLRP3 inflammasome in myofibroblasts (Boza et al., 2016), while lipopolysaccharide (LPS) and adenosine triphosphate (ATP) can be used experimentally to prime and activate the inflammasome, respectively (Boza et al., 2016; Cáceres et al., 2019). Currently, there is a lack of available therapies that directly target scar tissue accumulation in damaged organs. Accordingly, a better understanding of the molecular mechanism involved in myofibroblast NLRP3 inflammasomeinduced fibrosis progression may lead to improved and effective therapeutic targeting of NLRP3 inflammasome activation to treat fibrosis-induced end-organ damage and dysfunction.

The recombinant form of the peptide hormone relaxin, serelaxin (RLX), has emerged as a rapidly-acting anti-fibrotic therapy (Nistri et al., 2007; Du et al., 2010; Samuel et al., 2017), signaling via its cognate $\mathrm{G}$ protein-coupled receptor, relaxin family peptide receptor (RXFP)1 (Bathgate et al., 2013). Several lines of evidence have indirectly suggested that RLX may also inhibit the NLRP3 inflammasome to mediate its anti-fibrotic actions. First, RLX can suppress the pro-fibrotic influence of known inducers of myofibroblast NLPR3 inflammasome activity, including TGF- $\beta 1$ (Unemori and Amento, 1990; Mookerjee et al., 2009; Sarwar et al., 2015; Wang et al., 2016) and TLR-4 (Chen et al., 2017). RLX has been found to inhibit TGF- $\beta 1$ signal transduction at the level of intracellular Smad2, either through direct effects on cyclic guanosine monophosphate (cGMP) (Kocan et al., 2017) or by signaling through a RXFP1-extracellular signal-regulated kinases (ERK)-1/2 phosphorylation-neuronal nitric oxide (NO) synthase (nNOS)-NO-soluble guanylyl cyclase (sGC)-cGMP-dependent pathway (Mookerjee et al., 2009; Sarwar et al., 2015; Wang et al., 2016). This in turn results in the suppression of TGF- $\beta 1$-induced myofibroblast differentiation and myofibroblast-induced collagen production (the basis of fibrosis). Second, RLX has also been found to suppress both TGF- $\beta 1$ - and IL- $1 \beta$-mediated collagen synthesis and secretion in human dermal myofibroblasts (Unemori and Amento, 1990). Third, RLX was shown to suppress the proinflammatory and/or pro-fibrotic influence of IL- $1 \beta$ and IL-18 in vivo, in experimental models of renal ischemia/reperfusion injury (Collino et al., 2013) and atrial fibrillation (Beiert et al., 2017). Finally, RLX was found to attenuate myocardial ischemia/ reperfusion injury by inhibiting caspase- 1 activity (Valle Raleigh et al., 2017). However, caspase-1 activity can be modulated by several inflammasomes and no direct evidence was shown to link the findings obtained to the NLRP3 inflammasome in the latter study (Valle Raleigh et al., 2017).

We recently demonstrated in TGF- $\beta 1$-stimulated human cardiac myofibroblasts in vitro and in the left ventricle of mice subjected to isoproterenol-induced cardiomyopathy in vivo that RLX could inhibit the TGF- $\beta 1 / \mathrm{IL}-1 \beta / \mathrm{IL}-18$ axis via a RXFP1nNOS-TLR-4-NLRP3 inflammasome-dependent mechanism (Cáceres et al., 2019). While these findings confirmed for the first time that RLX could target inducers (TGF- $\beta 1$ and TLR- 4 ) of the myofibroblast NLRP3 inflammasome to mediate its antifibrotic actions, it was unclear whether RLX could also modulate the NLPR3 inflammasome itself to inhibit myofibroblast differentiation and collagen-I deposition. Hence, in this study, we extended our recent findings to further delineate whether RLX also modulated the myofibroblast NLRP3 inflammasome to mediate its anti-fibrotic actions on myofibroblast differentiation and collagen I deposition. This was addressed using a RXFP1expressing (Hossain et al., 2011; Chow et al., 2012) human dermal fibroblast (HDF) cell line subjected to siRNA-induced knock-down of specific components of NLRP3 inflammasome priming, NLRP3, ASC, or caspase-1. 


\section{MATERIALS AND METHODS}

\section{Materials}

The BJ3 human dermal fibroblast (HDF) cell line used in this study was kindly provided by William C. Hahn (Department of Medical Oncology, Dana-Farber Cancer Institute, Boston, MA). Recombinant human TGF- $\beta 1$ was obtained from In Vitro Technologies (\#240-B, Minneapolis, MN, United States). Recombinant human gene-2 (H2) relaxin was generously provided by Corthera Inc (San Mateo, CA, United States; a subsidiary of Novartis International AG, Basel, Switzerland). LPS (\#L2630) and ATP (\#A6419) were both obtained from SigmaAldrich (Vic, Australia).

\section{Culture of HDFs}

BJ3 HDFs were characterized as described before (Chow et al., 2012) and cultured in DMEM medium containing 17\% medium199, 15\% fetal bovine serum (FBS), $1 \%$ penicillin $(50 \mathrm{U} / \mathrm{ml}) /$ streptomycin $(50 \mu \mathrm{g} / \mathrm{ml}), 1 \%$ L-glutamine $(200 \mathrm{mM})$ and $2.2 \%$ HEPES buffer (all obtained from Life Technologies, Mulgrave, Vic, Australia). HDFs were cultured in $\mathrm{T}-175 \mathrm{~cm}^{2}$ flasks at $37^{\circ} \mathrm{C}$, passaged when $80 \%-90 \%$ confluent, and used between passages 10-14 for all of the outlined studies. At these passages, these cells were shown to express RXFP1 (Chow et al., 2012).

\section{Treatment of WT Human Dermal Myofibroblasts (HDMFs)}

Wild-type (WT) BJ3 HDFs were seeded at a density of $1-1.5 \times 10^{5}$ cells/well in 12-well plates and treated with TGF- $\beta 1$ (T, $5 \mathrm{ng} / \mathrm{ml})$ to stimulate the differentiation of these cells into myofibroblasts (HDMFs). Sub-groups of T-stimulated HDMFs were also treated with the NLRP3 inflammasome primer, LPS (L, $100 \mathrm{ng} / \mathrm{ml})$ and activator, ATP $(\mathrm{A}, 5 \mathrm{mM})(\mathrm{T}+\mathrm{L}+\mathrm{A})$ to ensure activation of the inflammasome on myofibroblasts. As a control, separate subgroups of cells were stimulated with $\mathrm{L}+\mathrm{A}$ (without $\mathrm{T}$ ), to determine the effects of $\mathrm{L}+\mathrm{A}$ stimulation on HDFs.

Sub-groups of $\mathrm{T}$ - or $\mathrm{T}+\mathrm{L}+\mathrm{A}$-treated HDMFs were then either left untreated or treated with RLX (RLX or R, $16.8 \mathrm{nM}$, which is equivalent to $100 \mathrm{ng} / \mathrm{ml}$ ) for $8 \mathrm{~h}$ [when NLRP3 inflammasome activation is optimally measured (Boza et al., 2016)] or for $72 h$ [when changes in TGF- $\beta 1$-induced myofibroblast differentiation and collagen I deposition could be measured (Samuel et al., 2004)]. The doses of each factor used were previously reported in studies that induced expression of the NLRP3 inflammasome (Boza et al., 2016) and determined the therapeutic effects of RLX on the myofibroblast NLRP3 inflammasome (Cáceres et al., 2019). Measures of NLRP3 priming [NLRP3, ASC and (pro)caspase-1] and activity (IL-1 $\beta$ and IL-18) were detected after $8 h$, while changes in NLRP3 priming, myofibroblast differentiation ( $\alpha$-SMA expression), and collagen I deposition (as measures of fibrosis) were detected following $72 h$ in $\mathrm{T}-, \mathrm{T}+\mathrm{R}-, \mathrm{T}+\mathrm{L}+\mathrm{A}_{-}$, and $\mathrm{T}+\mathrm{L}+\mathrm{A}+\mathrm{R}$-treated HDMFs. These studies were conducted 6-8 separate times in duplicate.

\section{HDMF siRNA Transfection Studies}

BJ3 HDMFs were seeded at a density of $1-1.5 \times 10^{5}$ cells/well in 12 -well plates, as described above. Short-interfering-RNA
(siRNA) knock-down technology was used to downregulate the expression of specific components of NLRP3 inflammasome priming, compared to control siRNA, in transfected cells. Lipofectamine RNAiMax transfection reagent (\#13778030; Life Technologies), Opti-MEM reduced serum medium (\#11058021; Life Technologies) and four separate individual siRNAs-one control siRNA (\#sc-37007), and three siRNAs each targeting NLRP3 (\#sc-45469), ASC (\#37281), or caspase-1 (\#29235) were prepared separately (all purchased from Santa Cruz Biotechnology, Dallas, TX, United States). Lipofectamine RNAiMax was diluted 1:100 in Opti-MEM reduced serum medium and left to incubate for 30 mins at room temperature (RT). Each of the siRNAs were also individually diluted 1:100 in Opti-MEM medium in separate tubes and left to incubate for 30 min at RT. The Lipofectamine RNAiMax/Opti-MEM mixture was then split into separate tubes and the siRNA/Opti-MEM mixtures were added to the Lipofectamine RNAiMax/OptiMEM mixtures. These were incubated for an additional $30 \mathrm{~min}$ prior to transfecting individual wells of HDFs to induce lipofectamine-mediated siRNA-knock-down of NLRP3, ASC or caspase- 1 for $24 h$. Separate wells of each plate were also treated with the CTL siRNA for the same time-period. The following day, the transfection medium was replaced with media and HDFs were left to rest for an additional day. The following day, transfected HDFs were treated in duplicate, as detailed below. In one set of experiments, HDFs were transfected with the CTL siRNA for $24 h$ before sub-groups of cells were further treated with $\mathrm{T}+\mathrm{L}+\mathrm{A}$ or $\mathrm{T}+\mathrm{L}+\mathrm{A}+\mathrm{R}$ for $72 h$. This was conducted to confirm that the effects RLX on measures of NLRP3 priming [NLRP3, ASC, and (pro)-caspase-1] and activity (IL-1 $\beta$ and IL$18)$ as well as fibrosis ( $\alpha$-SMA and collagen I) in cells transfected with the CTL siRNA reflected that observed following corresponding treatment of untransfected WT HDMFs. These studies were conducted 4-6 separate times in duplicate. In a separate set of experiments, HDFs separately transfected with the control or individual siRNAs that targeted NLRP3, ASC, or caspase-1, were treated with $\mathrm{T}$ or $\mathrm{T}+\mathrm{L}+\mathrm{A}$ for $8 h$ or $72 h$, to confirm knock-down of the individual NLRP3 inflammasome components. The siRNA-induced knock-down efficiency of each siRNA was determined by measuring the protein expression levels of NLRP3, ASC and (pro)-caspase-1, respectively. These studies were conducted 4-6 separate times in duplicate. In a final set of experiments, HDFs individually transfected with NLRP3 siRNA, ASC siRNA, caspase-1 siRNA or control siRNA for $24 h$, were then treated with $\mathrm{T}+\mathrm{L}+\mathrm{A}$ or $\mathrm{T}+\mathrm{L}+\mathrm{A}+\mathrm{R}$ for $72 h$ to determine which component(s) of the myofibroblast NLRP3 inflammasome RLX required an interaction with to mediate its inhibitory effects on myofibroblast differentiation and collagen I deposition. These studies were conducted 4-6 separate times in duplicate.

\section{Immunocytochemistry}

BJ3 HDFs (1-1.5x10 /well) were plated and grown on poly-Dlysine-coated coverslips in 12-well plates for $24 h$. BJ3 HDFs were then treated with $\mathrm{T}+\mathrm{L}+\mathrm{A}$ in the absence or presence of RLX for $8 h$ (to study the extent of NLRP3 colocalisation with caspase-1), and separately, for $72 h$ (to induce expression of $\alpha$-SMA, as a 
measure of myofibroblast differentiation). At the end of treatment, in each case, BJ3 HDMFs were washed once with warmed PBS, fixed for $10 \mathrm{~min}$ in $4 \%$ PFA, and washed once again in warmed PBS, prior to staining. Treated BJ3 HDMFs were then blocked with $10 \%$ normal goat serum containing $0.2 \%$ triton $\mathrm{X}$ 100 for $30 \mathrm{~min}$, and then incubated overnight in either goat polyclonal anti-NLRP3 (ab4207; 1:500 dilution; Abcam, Vic, Australia), rabbit polyclonal anti-caspase-1 (ab1872; 1:750 dilution; Abcam, Vic, Australia), or rabbit polyclonal anti- $\alpha$ SMA (ab5694; 1:500 dilution; Abcam, Vic, Australia) primary antibodies. The following day, primary antibodies were then aspirated and treated BJ3 HDMFs were washed 3 times in warmed PBS prior to incubation in the appropriate Alexa Fluor 488 or 594 secondary antibody (Alexa Fluor 488 donkey anti-goat IgG; \#A11012; 1:1000 dilution; or Alexa Fluor 594 goat anti-rabbit IgG; \#A20185; 1:750 dilution; both purchased from Life Technologies, Vic, Australia). Antibody-stained BJ3 HDMFs were then washed 3 times in warmed PBS, and cells mounted with a drop of VECTASHIELD HardSet ${ }^{\mathrm{TM}}$ Mounting Medium containing DAPI to preserve fluorescence (Vector Laboratories; Meadowbrook, Qld, Australia) on each coverslip, following mounting of another coverslip and left to dry. $\mathrm{T}+\mathrm{L}+\mathrm{A}$-treated BJ3 HDMFs dual stained for NLRP3 and caspase-1 were assessed for the degree of NLRP3 and caspase- 1 co-localisation compared to $\mathrm{T}+\mathrm{L}+\mathrm{A}+\mathrm{R}$-treated $\mathrm{BJ} 3 \mathrm{HDMFs}$ in $\alpha$-SMA differentiated HDMFs. All stained slides were scanned by Monash Micro Imaging using a Leica DMi8 Dexter microscope, at 40x magnification. Five to six field of view were randomly captured and analyzed using ImageJ Software (ImageJ, United States) to examine differences in the extent of NLRP3 and caspase-1 colocalization in $\mathrm{T}+\mathrm{L}+\mathrm{A}$ - vs. $\mathrm{T}+\mathrm{L}+\mathrm{A}+\mathrm{R}$-treated HDMFs.

\section{Western Blotting}

Following the various treatment groups examined in wild-type HDMFs, as well as in cells transfected with the CTL or individual siRNAs to NLRP3, ASC or caspase-1, the media was removed and the cell layer from treatment groups were lifted and collected via incubation in accutase solution (500 $\mu \mathrm{l}$ per well; SigmaAldrich, Castle Hill, NSW, Australia) for $10 \mathrm{mins}$ at $37^{\circ} \mathrm{C}$. Protein was extracted from the cell layer in $20 \mu \mathrm{l}$ of $1 \mathrm{x}$ RIPA lysis buffer, prepared from supplementing a 10x RIPA buffer (\#9806) with PMSF (\#8553), phosphatase/protease inhibitor (\#5872) (all purchased from Cell Signalling, Danvers, MA, United States), and double-distilled water. The suspension was left to incubate in ice for $1 \mathrm{~h}$, followed by centrifugation at 1500 rpm for $10 \mathrm{~min}$ at $4^{\circ} \mathrm{C}$ to pellet any cellular debris, leaving protein in solution. The supernatant containing the protein was then transferred to a separate Eppendorf tube, which was subsequently stored at $-20^{\circ} \mathrm{C}$ until required for use. Equivalent volumes of protein from HDMF-cell layers were electrophoresed on mini-protean $4 \%-15 \%$ precast gels and analyzed by Western blotting using monoclonal antibodies to NLRP3 (\#15101; 1:1000 dilution; Cell Signaling Technology, Danvers, MA, United States), ASC (ab155970; 1:1000 dilution; Abcam, Cambridge, MA, United States), pro-caspase-1 (\#3866; 1:1000 dilution; Cell Signaling Technology), pro-IL-1 $\beta$ (MAB601-100; 1:1000 dilution; In Vitro Technologies, Noble Park North, Vic, Australia), pro-IL-18 (D043-3; 1:1000 dilution; In Vitro Technologies), and $\alpha$-SMA (M0851; 1:1000 dilution; Dako/ Agilent Technologies, Mulgrave, Vic, Australia); or a polyclonal antibody to collagen I (ab34710; 1:1000 dilution; Abcam). The equivalent loading of protein between samples was confirmed using a monoclonal antibody to the housekeeping protein, GAPDH (ab8245; 1:1000 dilution; Abcam). In each case, appropriate anti-mouse or anti-rabbit HRP conjugated secondary antibodies (Cell Signaling Technologies) were used, while blots were detected using the Clarity Western ECL substrate detection kit and quantified by densitometry with a ChemiDoc $^{\mathrm{TM}}$ MP Imaging System and Image Lab version 6.0 software (both from Bio-Rad Laboratories, Richmond, CA, United States). The density of each parameter was corrected for GAPDH protein levels, and then expressed relative to the indicated control group in each case, which was expressed as 1 in each case.

\section{Measurement of Caspase-1 and IL-1 $\beta$ Activity}

As caspase- 1 and IL-1 $\beta$ activity are difficult to measure at the cellular level (based on cell number) (Cáceres et al., 2019), BJ3 HDMFs were seeded at a density of $1-1.5 \times 10^{6}$ cells/well in 12 well plates. HDMFs were then transfected with CTL or caspase-1 siRNA for $24 \mathrm{~h}$. Transfected HDMFs were then treated either with $\mathrm{T}+\mathrm{L}+\mathrm{A}$ or $\mathrm{T}+\mathrm{L}+\mathrm{A}+\mathrm{R}$ for $8 \mathrm{~h}$. After $8 \mathrm{~h}$, the media from nine sets of duplicate samples $(18 \mathrm{ml})$ for each treatment group were pooled and concentrated down to $300 \mu \mathrm{l}$ (x60) using Amicon ${ }^{\circledR}$ Ultra-4 centrifugal filter units with a $10 \mathrm{kDa}$ cut-off (Merck Millipore, Vic, Australia). Concentrated media samples were then assayed in triplicate using a R\&D Systems Human IL-1 $\beta$ / IL-1F2 Quantikine ELISA and Human Caspase-1/ICE Quantikine ELISA Kits (In Vitro Technologies, Vic, Australia), according to manufacturer's instructions.

\section{Statistical Analysis}

All statistical analysis was performed using GraphPad Prism v7.0 (GraphPad Software Inc., CA, United States). All data was analysed via a one-way ANOVA with Neuman-Keuls post-hoc test for multiple comparisons between groups. In each case, results were expressed as the relative mean \pm SEM with a $\mathrm{p}$ value $<0.05$ considered statistically significant.

\section{RESULTS}

\section{Effects of RLX on NLRP3 Inflammasome Priming and Activation in Wild-Type HDMFs After $8 \mathrm{~h}$}

Wild-type (WT) BJ3 HDFs were stimulated with LPS+ATP (L $+\mathrm{A})$ or with TGF- $\beta 1(\mathrm{~T})$ to promote their differentiation into myofibroblasts (HDMFs) as well as $\mathrm{T}+\mathrm{L}+\mathrm{A}$ to promote the induction of the NLRP3 inflammasome in T-stimulated HDMFs for $8 \mathrm{~h}$ [the optimal time-point at which NLRP3 
inflammasome activity was increased in myofibroblasts (Boza et al., 2016)]. While L+A-stimulated HDFs did not undergo any marked changes in NLRP3 inflammasome components (NLRP3, ASC, pro-caspase-1; Supplementary Table 1); $\mathrm{T}+\mathrm{L}+\mathrm{A}-$ stimulated HDMFs had significantly increased protein expression of measures of NLRP3 inflammasome priming, NLRP3, ASC and pro-caspase-1 (by 45-120\%), as well as activity, pro-IL-1 $\beta$ (by $\sim 4$-fold) and pro-IL (by $\sim 28 \%$ ) after $8 \mathrm{~h}$, as determined by Western blotting (Figures 1A, B, all $\mathrm{p}<0.05$ vs. $\mathrm{T}$ alone). The $\mathrm{T}+\mathrm{L}+\mathrm{A}$-stimulated increase in cellular pro-caspase- 1 and pro-IL-1 $\beta$ protein expression correlated with a significant increase in maturely secreted caspase- 1 and IL- $1 \beta$ activity (Figure 1C, both $\mathrm{p}<0.01$ vs. $\mathrm{T}$ alone), as determined by ELISA analysis of concentrated media samples. RLX (R, $100 \mathrm{ng}$ / $\mathrm{ml}$ ) administration to $\mathrm{T}$ alone-stimulated HDMFs did not significantly affect any of these end-points measured. However, RLX administration to $\mathrm{T}+\mathrm{L}+\mathrm{A}$-stimulated HDMFs significantly inhibited NLRP3, ASC, pro-caspase-1, and pro-IL-18 protein expression as well as mature IL-1 $\beta$ activity to levels that were no longer different to that measured in $\mathrm{T}$ alone-stimulated cells (Figures 1B, C, all $\mathrm{p}<0.05$ vs. $\mathrm{T}+\mathrm{L}+\mathrm{A}$ ); and partially inhibited pro-IL-1 $\beta$ protein expression (by $\sim 63 \%$ ) and mature caspase- 1 levels (by $\sim 36 \%$ ) (Figures 1A, C, both $\mathrm{p}<0.05$ vs. $\mathrm{T}+\mathrm{L}+\mathrm{A}$; $\mathrm{p}<0.05$ vs. $\mathrm{T}$ alone). RLX administration to $\mathrm{T}+\mathrm{L}+\mathrm{A}$-stimulated $\mathrm{WT}$ HDMFs also reduced the degree of co-localization between NLRP3 and caspase- 1 in these cells, compared to T+L+Astimulated cells alone (Figure 1D).

\section{Effects of RLX on NLRP3 Inflammasome Priming/Activation and Fibrosis in WT HDMFs After $72 \mathrm{~h}$}

WT BJ3 HDFs were separately stimulated with $\mathrm{L}+\mathrm{A}$, $\mathrm{T}$ or $\mathrm{T}+\mathrm{L}+\mathrm{A}$ for $72 \mathrm{~h}$ (representing the time point at which changes in markers of fibrosis could be measured from myofibroblasts), and $\mathrm{T}$ or $\mathrm{T}+\mathrm{L}+\mathrm{A}$-stimulated HDMFs co-treated with RLX (R) over the same time-period. Once again, $\mathrm{L}+\mathrm{A}$-stimulation of HDF failed to induce any marked changes in NLRP3 inflammasome components (NLRP3, ASC, pro-caspase-1; Supplementary Table 1). On the other hand, $\mathrm{T}+\mathrm{L}+\mathrm{A}$-stimulated HDMFs had significantly increased protein expression of NLRP3, ASC, procaspase-1 (by $\sim 38-155 \%$ ), pro-IL-1 $\beta$ (by $\sim 3.5$-fold), and pro-IL18 (by $\sim 125 \%$ ), which corresponded to a modest but significant increase in $\alpha$-SMA (by $\sim 20 \%$ ) and collagen I (by $\sim 30 \%$ ) protein expression (Figure 2, all $\mathrm{p}<0.05$ vs. T alone) after $72 \mathrm{~h}$. RLX significantly inhibited $\alpha$-SMA and collagen I protein expression in $\mathrm{T}$ alone-stimulated cells (Figure 2, by $\sim 15-21 \%$; both $\mathrm{p}<0.05$ vs. $T$ alone) in the absence of having any effects on measures of the NLRP3 inflammasome. However, RLX fully inhibited the T $+\mathrm{L}+\mathrm{A}$-stimulated increase in NLRP3, ASC and pro-caspase-1 (Figure 2, all $\mathrm{p}<0.05$ vs. $\mathrm{T}+\mathrm{L}+\mathrm{A}$ ); partially inhibited the $\mathrm{T}+\mathrm{L}+\mathrm{A}-$ stimulated increase in pro-IL-1 $\beta$ (Figure 2, by $\sim 70 \% ; \mathrm{p}<0.01$ vs. $\mathrm{T}+\mathrm{L}+\mathrm{A} ; \mathrm{p}<0.05$ vs. $\mathrm{T}$ alone); and further inhibited the $\mathrm{T}+\mathrm{L}+\mathrm{A}-$ stimulated increase in levels of $\alpha$-SMA and collagen I protein expression (Figure 2, both $\mathrm{p}<0.01$ vs. $\mathrm{T}+\mathrm{L}+\mathrm{A}$ ) to those that were measured from RLX treatment of $\mathrm{T}$ alone-stimulated cells (Figure 2).

\section{Validation of Lipofectamine-siRNA- Mediated Knock-Down of NLRP3 Inflammasome Components in WT HDMFs}

We first determined whether the control (CTL) siRNA obtained had any effects on the NLRP3 inflammasome-inhibitory and anti-fibrotic effects of RLX in HDMFs. Consistent with the data obtained from RLX-treated WT HDMFs after 72 h (Figure 2), RLX treatment of CTL siRNA transfected-HDMFs stimulated with $\mathrm{T}+\mathrm{L}+\mathrm{A}$ (CTL siRNA+T+L+A+R), significantly inhibited protein expression levels of NLRP3, ASC, pro-caspase-1, pro-IL$1 \beta$, pro-IL-18, $\alpha$-SMA and collagen I (Figures $3 \mathbf{A}, \mathbf{B}$, all $\mathrm{p}<0.05$ vs. CTL siRNA+T+L+A); as well as mature levels of caspase-1 and IL- $1 \beta$ activity (Figure $3 \mathrm{C}$, both $\mathrm{p}<0.05$ vs. CTL siRNA $+\mathrm{T}+\mathrm{L}$ $+\mathrm{A})$ after $72 \mathrm{~h}$. These findings indicated that the anti-fibrotic effects of RLX in T+L+A-stimulated HDMFs were not altered by the presence of the CTL siRNA.

We then examined the siRNA-induced knock-down efficiency of NLRP3, ASC or caspase-1 in T- and T+L+Astimulated HDMFs after $8 \mathrm{~h}$ and $72 \mathrm{~h}$, in comparison to the CTL siRNA, which should not have induced any knock-down of the end-points measured (Figure 4). Compared to CTL siRNA transfected-HDMFs that were stimulated with $\mathrm{T}$ alone, CTL siRNA transfected-HDMFs stimulated with $\mathrm{T}+\mathrm{L}+\mathrm{A}$ expressed significantly increased NLRP3, ASC, and pro-caspase- 1 after $8 \mathrm{~h}$ (by $~ 50 \%-75 \%$ ) and $72 \mathrm{~h}$ (by $\sim 40 \%-55 \%$ ) (Figure 4, all $\mathrm{p}<0.05$ vs. CTL siRNA+T alone). siRNA-induced knock-down of NLRP3 (NLRP3 siRNA+T+L+A) or caspase-1 (caspase-1 siRNA+T+L $+\mathrm{A}$ ) did not reduce NLRP3 or caspase-1 expression, respectively after $8 \mathrm{~h}$, but induced complete knock-down of NLRP3 or caspase-1 expression, respectively after $72 \mathrm{~h}$ (Figure 4, both $\mathrm{p}<0.05$ vs. CTL siRNA+T+L+A; no difference to CTL siRNA+T alone). In contrast, siRNA-induced knock-down of ASC (ASC siRNA $+\mathrm{T}+\mathrm{L}+\mathrm{A}$ ) significantly reduced ASC expression from $\mathrm{T}+\mathrm{L}$ + A-stimulated HDMFs at $8 \mathrm{~h}$ and to a greater extent at $72 \mathrm{~h}$ (Figure 4, both $\mathrm{p}<0.05$ vs. CTL siRNA $+\mathrm{T}+\mathrm{L}+\mathrm{A}$; difference to CTL siRNA+T alone). These findings confirmed the complete siRNA-induced knock-down of NLRP3, ASC, or caspase-1 from $\mathrm{T}+\mathrm{L}+\mathrm{A}$-stimulated HDMFs after $72 \mathrm{~h}$.

\section{The Effects of RLX on the Myofibroblast NLRP3 Inflammasome in the Absence or Presence of siRNA Treatment}

Following establishment of siRNA-mediated knock-down of NLRP3, ASC or caspase-1 in HDMFs, we next examined whether RLX modulated the myofibroblast NLRP3 inflammasome to mediate its anti-fibrotic actions. The stimulation of HDFs with $\mathrm{T}+\mathrm{L}+\mathrm{A}$ in the presence of CTL siRNA (black bars), NLRP3 siRNA (blue bars), ASC siRNA (purple bars), and caspase-1 siRNA (green bars), had comparable effects on measures of NLRP3 priming and activity as well as fibrosis (Figures 5A, B). In contrast, the administration of RLX to CTL siRNA transfected and $\mathrm{T}+\mathrm{L}+\mathrm{A}$-stimulated HDMFs significantly downregulated measures of NLRP3 priming and activity, and fibrosis (Figures 5A, B), which was consistent with the effects of RLX shown in WT HDMFs (Figures 2A, B; in the absence of siRNA). These inhibitory effects of RLX were 
A
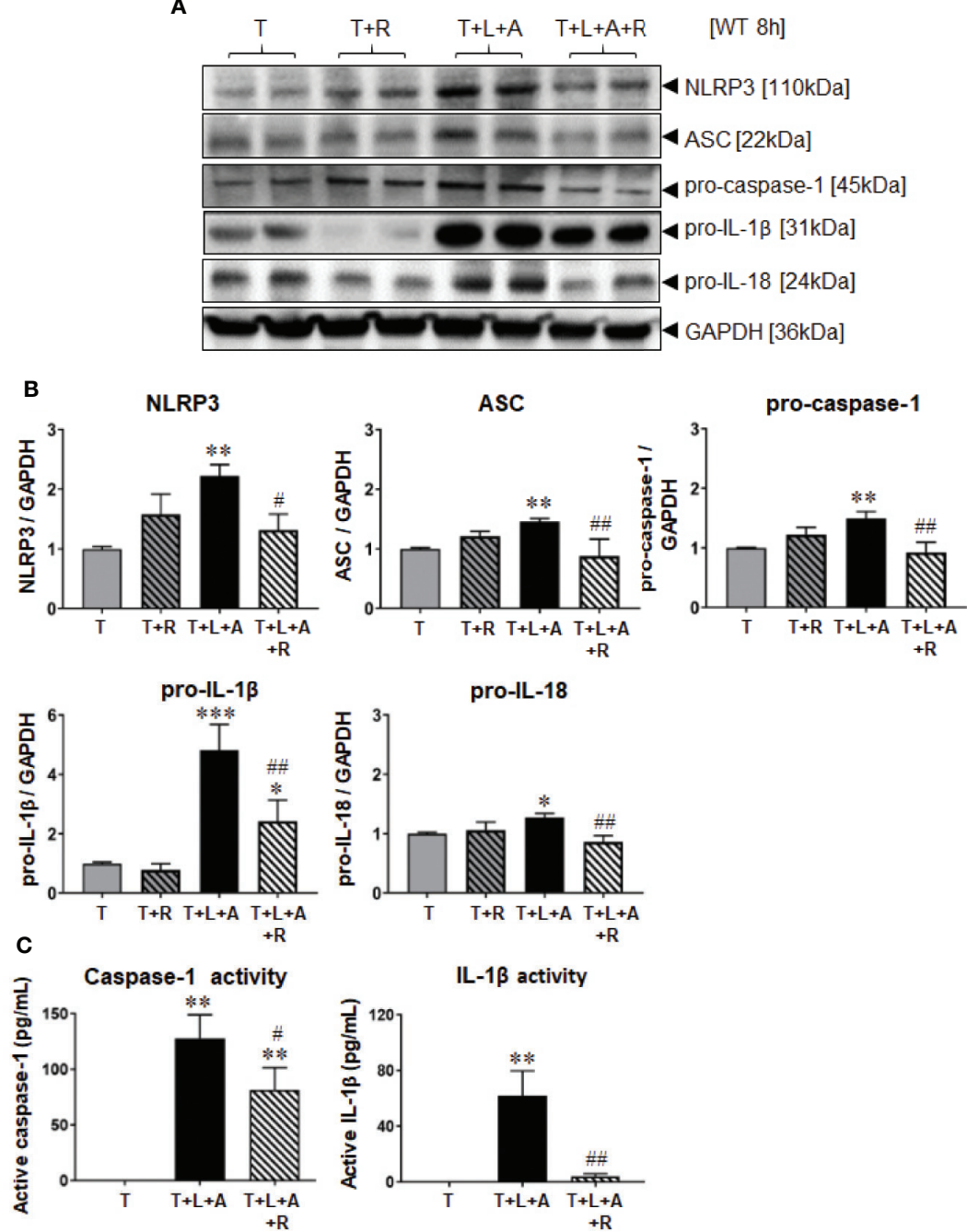

IL-1 $\beta$ activity
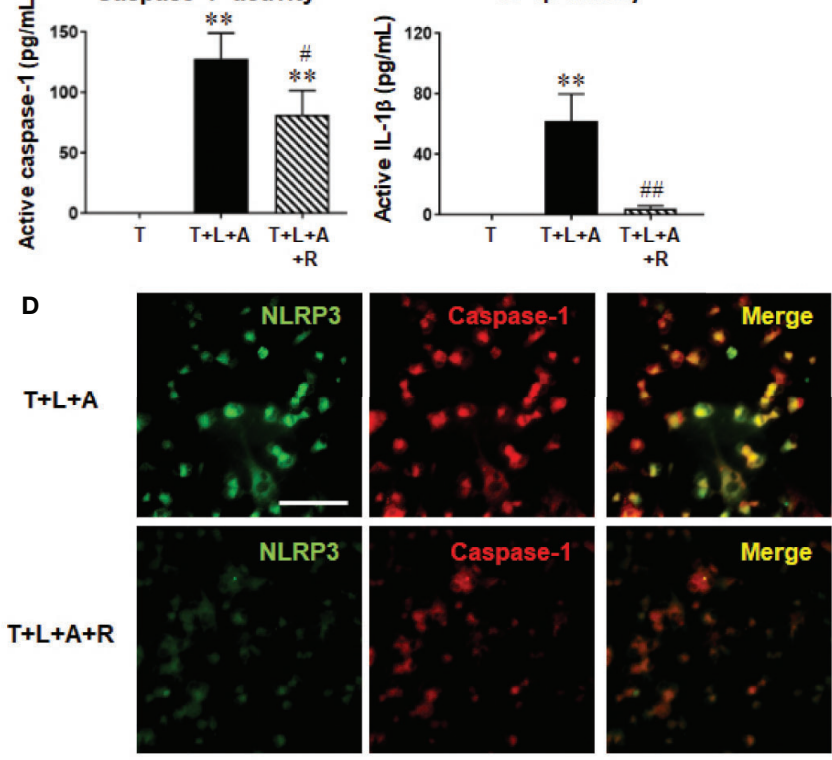

FIGURE 1 | The effects of RLX on NLRP3 inflammasome priming and activity in T- and T+L+A-stimulated WT BJ3 HDMFs after 8 h. (A) Representative Western blots of NLRP3, ASC, pro-caspase-1, pro-IL-1 $\beta$, and pro-IL-18 protein expression from HDMFs stimulated with TGF- $\beta 1$ (T; 5 ng/ml), T+R (RLX; 100 ng/ml), T+L (LPS; $100 \mathrm{ng} / \mathrm{ml})+\mathrm{A}($ ATP; $5 \mathrm{mM}$ ), or $\mathrm{T}+\mathrm{L}+\mathrm{A}+\mathrm{R}$ over $8 \mathrm{~h}$. (B) Also shown are the mean \pm SEM of each end-point measured from each treatment group studied, corrected for GAPDH loading and expressed relative to the value in the T-treated group, which was expressed as 1 in each case; from $n=6-8$ separate experiments conducted in duplicate. (C) Additionally shown are the mean \pm SEM levels of mature, secreted active caspase-1 (pg/ml) and active IL-1 $\beta$ (pg/ml) activity detected from the concentrated media of $\mathrm{T}$-, $\mathrm{T}+\mathrm{L}+\mathrm{A}$ - and $\mathrm{T}+\mathrm{L}+\mathrm{A}+\mathrm{R}$-treated HDMFs after $8 \mathrm{~h} .{ }^{*} \mathrm{p}<0.05,{ }^{\star \star} \mathrm{p}<0.01,{ }^{\star \star \star} \mathrm{p}<0.001$ vs. T-alone treated group; ${ }^{\#} \mathrm{p}<0.05$, ${ }^{\# \#} \mathrm{p}<0.01$ vs. $T+L+A$-treated group. (D) Representative images of NLRP3, caspase-1 and co-localization of NLRP3 and caspase-1 in $T+L+A-v s$. $T+L+A+R-t r e a t e d ~ H D M F s$ confirm the presence of the NLRP3 inflammasome in the HDMFs studied, and the ability of RLX to inhibit the individual and co-localized components of the inflammasome. Scale bar; $100 \mu \mathrm{m}$. 
A
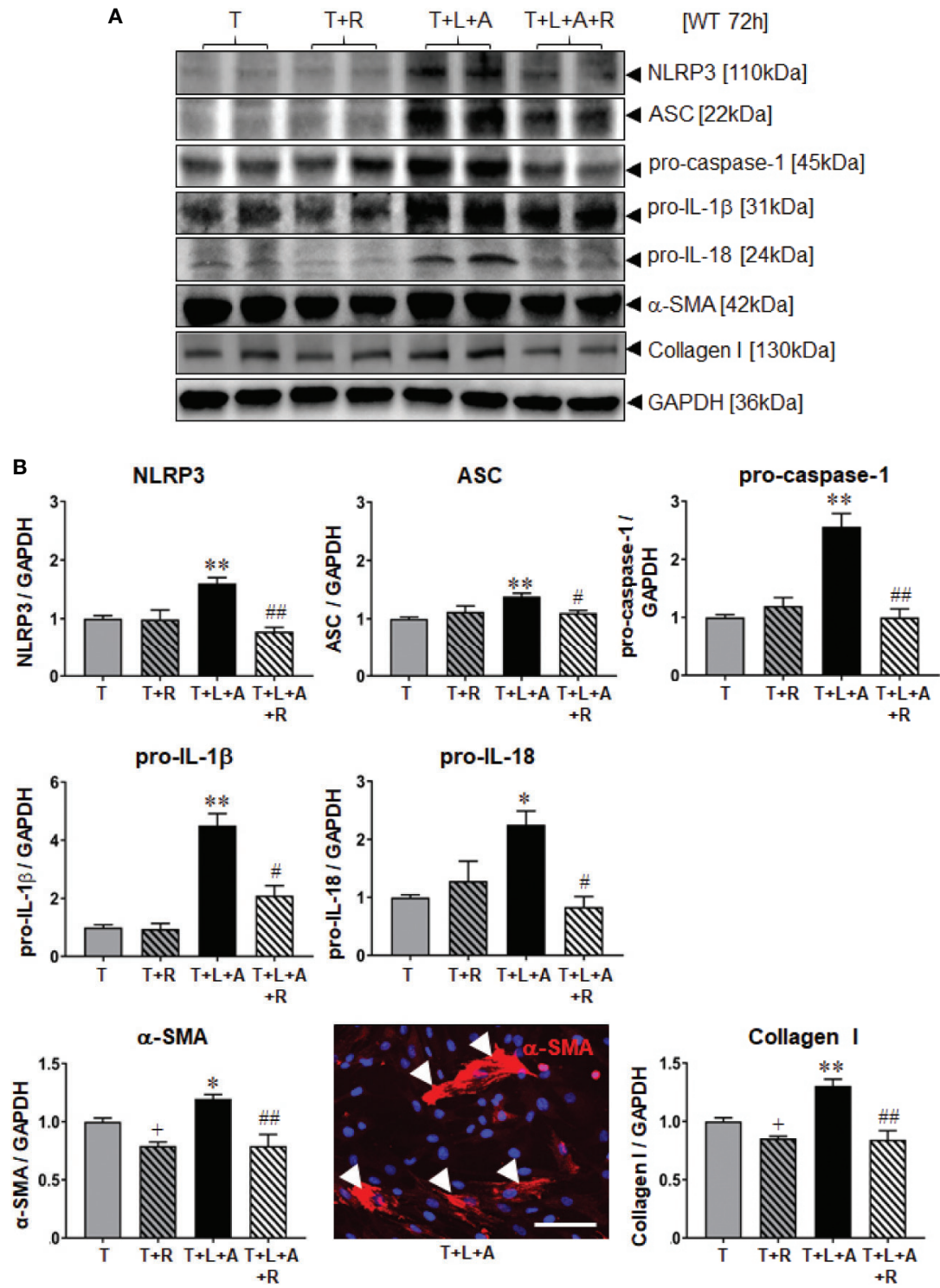

FIGURE 2 | The effects of RLX on NLRP3 inflammasome priming and activity, and measures of fibrosis in T-and T+L+A-stimulated WT BJ3 HDMFs after $72 \mathrm{~h}$. (A) Representative Western blots of NLRP3, ASC, pro-caspase-1, pro-IL-1 $\beta$ and pro-IL-18, $\alpha$-SMA (used as a marker of myofibroblast differentiation), and collagen I protein expression from HDMFs stimulated with TGF- $\beta 1$ (T; $5 \mathrm{ng} / \mathrm{ml})$, T+R (RLX; $100 \mathrm{ng} / \mathrm{ml}), T+L(L P S ; 100 \mathrm{ng} / \mathrm{ml})+A(A T P ; 5 \mathrm{mM})$, or T+L+A+R over 72 h. (B) Also shown are the mean \pm SEM of each end-point measured from each treatment group studied, corrected for GAPDH loading and expressed relative to the value in the T-treated group, which was expressed as 1 in each case; from $n=6-8$ separate experiments conducted in duplicate. ${ }^{*} p<0.05$, ${ }^{* *} p<0.01$ vs. T-alone treated group; ${ }^{\#} \mathrm{p}<0.05,{ }^{\# \#} \mathrm{p}<0.01$ vs. $T+\mathrm{L}+\mathrm{A}$-treated group. Immunofluoresence (red) staining of $\alpha$-SMA in stress fibres (indicated by white arrows) in $T+L+A-s t i m u l a t e d ~ H D M F s$ confirmed that these stimulated cells were myofibroblasts after $72 \mathrm{~h}$ of culture. 4',6-diamidino-2-phenylindole (DAPI; blue) staining was used to detect cell nuclei. Scale bar; $100 \mu \mathrm{m}$.

unaffected by the presence of siRNA targeting NLRP3 or ASC (Figures 5A, B). These findings suggested that RLX did not modulate NLRP3 or ASC within the myofibroblast NLRP3 inflammasome to inhibit pro-IL-1 $\beta$ and pro-IL-18. However, only in the presence of siRNA targeting caspase-1 (where caspase-1 was knocked-down prior to stimulation with $\mathrm{T}+\mathrm{L}+\mathrm{A}$ $\pm \mathrm{R}$ ) was the ability of RLX to downregulate caspase-1 (but not NLRP3 or ASC), pro-IL-1 $\beta$, pro-IL-18, $\alpha$-SMA, and collagen-I, completely abrogated, indicating that RLX was able to modulate caspase-1 within the myofibroblast NLRP3 inflammasome to mediate its anti-fibrotic effects (Figures 5A, B). To further consolidate these results, we measured the levels of mature caspase and IL-1 $\beta$ activity in caspase-1 siRNA transfected HDMFs treated with $\mathrm{T}+\mathrm{L}+\mathrm{A}$ and also separately with $\mathrm{T}+\mathrm{L}+\mathrm{A}$ $+\mathrm{R}$ (Figure 5C). In the presence of siRNA targeting caspase-1, the ability of RLX to downregulate caspase- 1 and IL- $1 \beta$ activity was suppressed. These findings confirmed that RLX could modulate the myofibroblast NLRP3 inflammasome at the level 

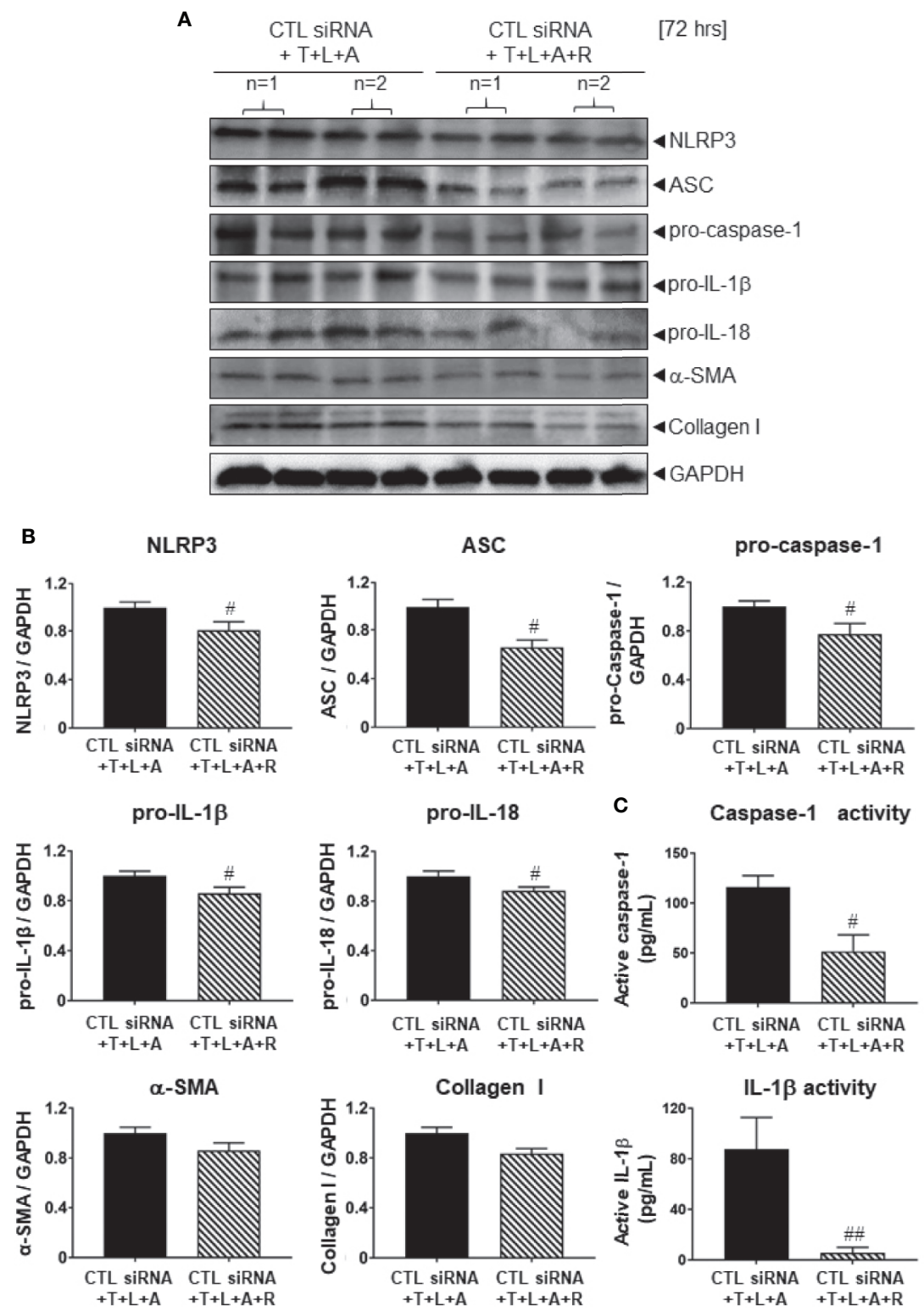

FIGURE 3 | The effects of RLX on Lipofectamine-induced CTL siRNA transfection in NLRP3 inflammasome activated BJ3 HDMFs after 72 h. (A) Representative Western blots of NLRP3, ASC, pro-caspase-1, pro-IL-1 $\beta$, pro-IL-18, $\alpha-S M A$, and collagen-I protein expression in $H D M F s$ treated with $C T L$ siRNA $\pm T+L+A \pm R$ treatment after $72 \mathrm{~h}$. (B) Also shown are the mean \pm SEM of each end-point measured from each treatment group studied, corrected for GAPDH loading and expressed relative to the value in the CTL siRNA+T+L+A-treated HDMF group, which was expressed as 1 in each case; from $n=4-6$ separate experiments conducted in duplicate. ${ }^{\#} \mathrm{p}<0.0{ }^{\# \#} \mathrm{p}<0.01 \mathrm{vs}$. CTL siRNA+T+L+A-treated group. (C) Additionally shown are the mean $\pm \mathrm{SEM}$ levels of active caspase-1 (pg/ml) (top panel) and active $\mathrm{IL}-1 \beta$ (pg/ml) (bottom panel) in CTL siRNA transfected HDFs treated with $\mathrm{T}+\mathrm{L}+\mathrm{A}$ or $\mathrm{T}+\mathrm{L}+\mathrm{A}+\mathrm{R}$ after $72 \mathrm{~h}$.

of caspase-1 to inhibit pro-IL-1 $\beta$, pro-IL-18, myofibroblast differentiation, and myofibroblast-induced collagen I deposition.

\section{DISCUSSION}

RLX has emerged as a rapidly-acting anti-fibrotic therapy, and at least at the experimental level, as a more efficacious antifibrotic compared to the ACE inhibitor, enalapril (Samuel et al.,
2014). It was previously shown that RLX mediated its antifibrotic effects through direct (Kocan et al., 2017) or indirect (Mookerjee et al., 2009; Wang et al., 2016) activation of cGMP, the latter involving nNOS-NO-sGC-cGMP signaling, to inhibit TGF- $\beta 1$ signal transduction at the level of intracellular Smad2 (Mookerjee et al., 2009; Samuel et al., 2014; Wang et al., 2016; Kocan et al., 2017) and/or Smad3 (Kocan et al., 2017) in myofibroblasts. This consistently resulted in the RLX-induced inhibition of the pro-fibrotic influence of TGF- $\beta 1$ on 
A

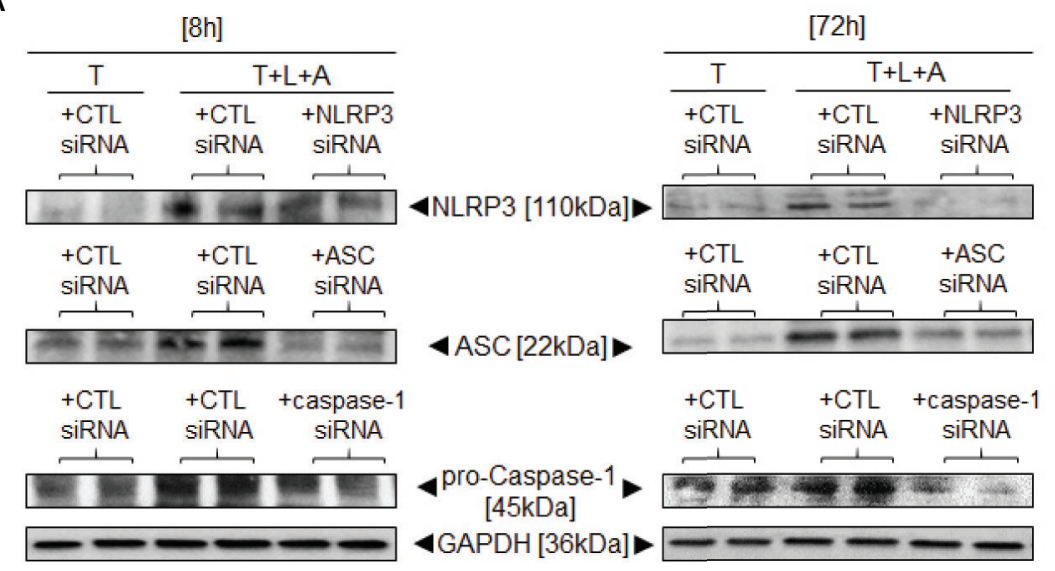

B
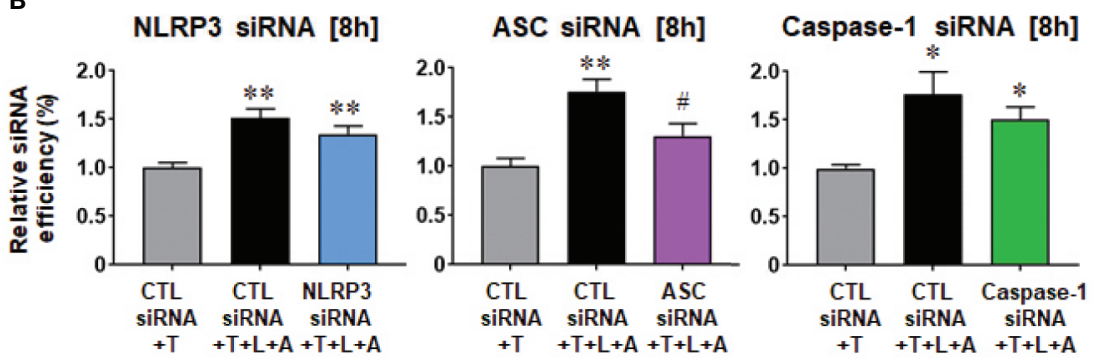

ASC siRNA [72h]
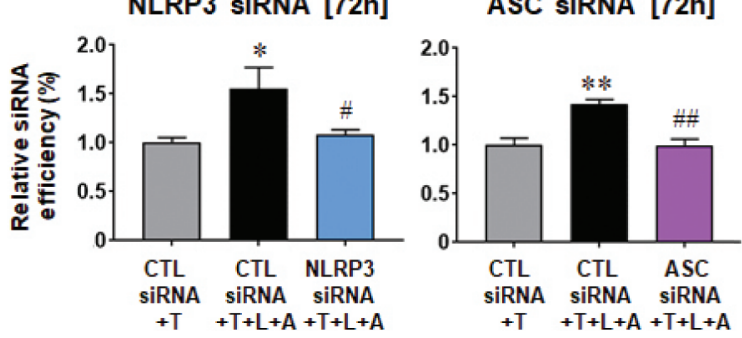

\section{Caspase-1 siRNA [72h]}

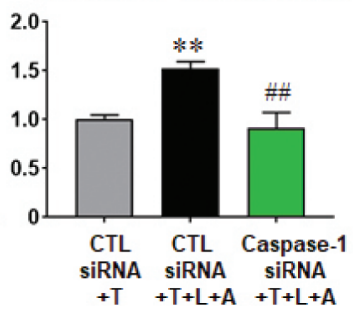

FIGURE 4 | Lipofectamine-mediated siRNA-induced knock-down of NLRP3, ASC or pro-caspase-1 after 8 or 72 h in BJ3 HDMFs. (A) Representative Western blots of NLRP3, ASC and pro-caspase-1 protein expression in HDMFs treated with CTL siRNA+T, CTL siRNA+T+L+A, or NLRP3 siRNA+T+L+A, ASC siRNA+T+L $+\mathrm{A}$, or caspase-1 siRNA+T+L+A, respectively, after 8 or $72 \mathrm{~h}$ (where siRNA were added for only $24 \mathrm{~h}$ for the $72 \mathrm{~h}$ end-points measured). (B) Also shown are the mean \pm SEM of the transfection efficiency of each siRNA used against its target protein, at each time-point studied. Each measure was corrected for GAPDH loading and expressed relative to the value in the CTL siRNA+T-treated group, which was expressed as 1 in each case; from $n=4-6$ separate experiments conducted in duplicate. ${ }^{*} \mathrm{p}<0.05,{ }^{* *} \mathrm{p}<0.01$ vs. CTL siRNA+T-treated group; ${ }^{\#} \mathrm{p}<0.05,{ }^{\# \#} \mathrm{p}<0.01$ vs. CTL siRNA+T+L+A-treated group.

myofibroblast differentiation and myofibroblast-induced collagen deposition, in the absence of RLX having any direct effects on collagen (Unemori et al., 1996; Samuel et al., 2004). Given that the inflammatory cytokine, IL-1 $\beta$, can promote fibrosis progression through an autocrine loop with the TGF$\beta 1 /$ Smad3 axis (Bonniaud et al., 2005; Aoki et al., 2006), and that RLX was found to inhibit collagen synthesis from either IL$1 \beta$ or TGF- $\beta 1$-stimulated HDMFs (Unemori and Amento, 1990 ), our recent attention turned to how RLX disrupted the TGF- $\beta 1 /$ IL- $1 \beta$ interaction. As RLX was also found to inhibit renal fibrosis through a TLR-4-dependent mechanism (Chen et al., 2017), which is a known inducer of the myofibroblast NLRP3 inflammasome (Boza et al., 2016), and IL-1 $\beta$ along with
IL-18 can be produced by this inflammasome (Artlett and Thacker, 2015), we investigated whether RLX targeted TGF$\beta 1$ and TLR-4-induced NLRP3 inflammasome activity in myofibroblasts to mediate its anti-fibrotic actions.

Our recent findings revealed that RLX signaled through a RXFP1-nNOS-TLR-4-dependent mechanism in human cardiac myofibroblasts in vitro and in a murine model of isoproterenolinduced cardiomyopathy in vivo to inhibit measures of myofibroblast NLRP3 inflammasome priming and activity, as well as myofibroblast differentiation and collagen I deposition (Cáceres et al., 2019). These findings extended the abovementioned studies in demonstrating that RLX could ameliorate NLRP3 inflammasome activity in myofibroblasts to inhibit the 
A

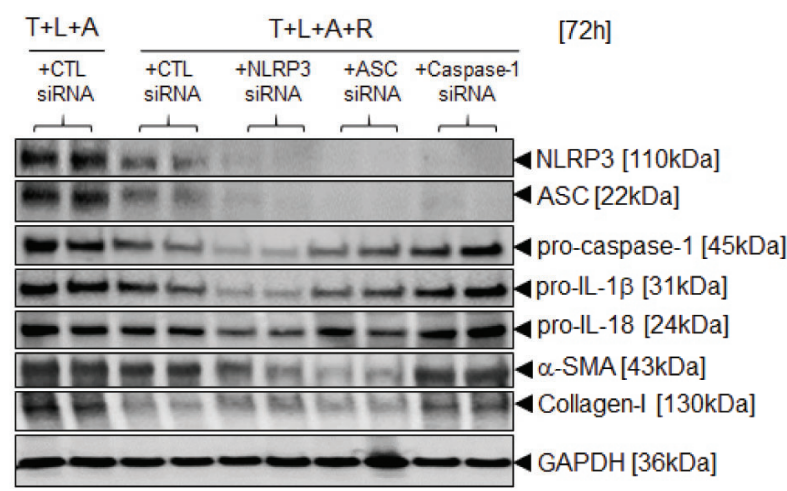

B NLRP3

ASC

pro-caspase-1
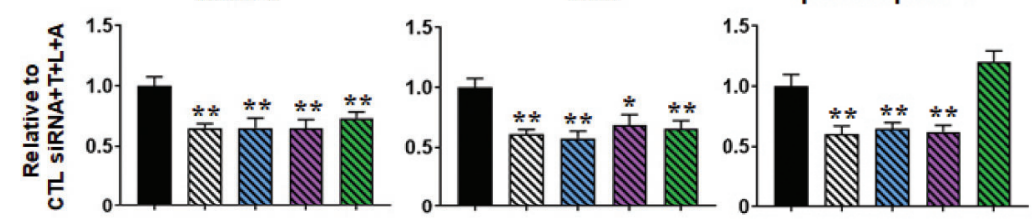

pro-IL-1ß

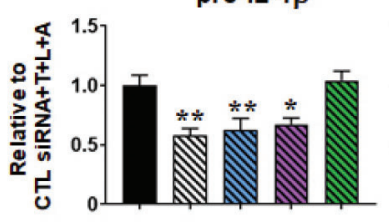

pro-IL-18

c
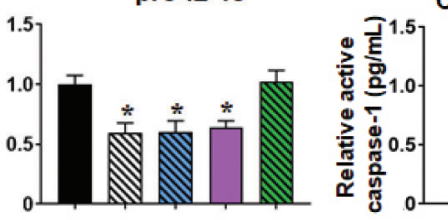

Caspase-1 activity

$\alpha-$ SMA

Collagen I

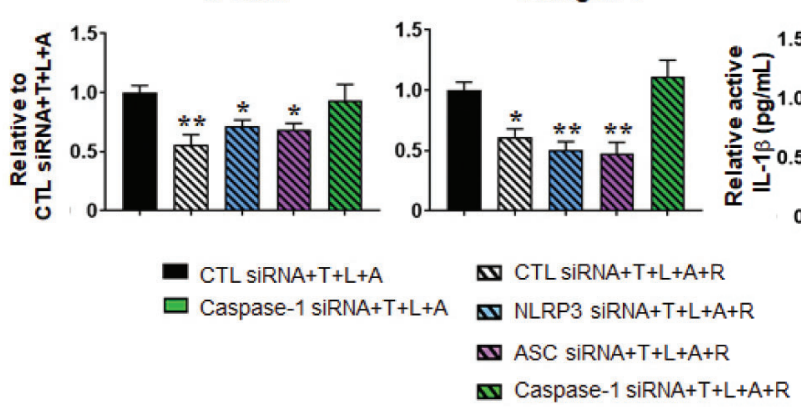

FIGURE 5 | The effects of RLX in siRNA-transfected and NLRP3 inflammasome activated BJ3 HDMFs after $72 \mathrm{~h}$. (A) Representative Western blots of NLRP3, ASC, pro-caspase-1, pro-IL-1 $\beta$, pro-IL-18, $\alpha$-SMA, and collagen I protein expression in HDMFs transfected with CTL siRNA for $24 \mathrm{~h}$ and subsequently stimulated with T $+\mathrm{L}+\mathrm{A} \pm \mathrm{R}$ for $72 \mathrm{~h}$; or NLRP3 siRNA, ASC siRNA, or caspase-1 siRNA for $24 \mathrm{~h}$ and subsequently stimulated with $\mathrm{T}+\mathrm{L}+\mathrm{A}+\mathrm{R}$ for $72 \mathrm{~h}$. (B) Also shown are the mean \pm SEM of protein expression levels of each end-point measured, corrected for GAPDH loading and expressed relative to the value in the CTL siRNA+T+L+A-treated group; which was expressed as 1 in each case; from $n=4-6$ separate experiments conducted in duplicate. ${ }^{*} p<0.05,{ }^{* *} p<0.01$ vs. the $C T L$ siRNA+T+L+A-treated group. (C) Additionally shown are the relative mean \pm SEM levels of active caspase- 1 (pg/ml) (top panel) and active IL-1 13 (pg/ml) (bottom panel) in caspase-1 siRNA transfected HDFs treated with $\mathrm{T}+\mathrm{L}+\mathrm{A}$ or $\mathrm{T}+\mathrm{L}+\mathrm{A}+\mathrm{R}$ after $72 \mathrm{~h}$.

pro-fibrotic interaction between TGF- $\beta 1$, TLR-4 and IL-1 $\beta /$ IL-18 (Figure 6) on myofibroblast differentiation and myofibroblastinduced interstitial collagen deposition. Furthermore, they somewhat confirmed previous reports which showed that RLX could inhibit the pro-inflammatory and pro-fibrotic effects of IL$1 \beta$ and IL-18 in vivo, but which did not investigate NLRP3 inflammasome activity in the experimental models studied. Despite these novel findings, it remained unclear as to whether
RLX modulated the myofibroblast NLRP3 inflammasome to mediate its anti-fibrotic effects or was only able to indirectly regulating the inflammasome at the level of the TGF- $\beta 1$-TLR-4 axis on cardiac myofibroblasts (Boza et al., 2016). The findings from this study, now show for the first time, that RLX can directly modulate the myofibroblast NLRP3 inflammasome at the level of caspase- 1 to mediate its anti-fibrotic actions, as the ability of RLX to inhibit pro-IL-1 $\beta$, pro-IL-18, myofibroblast differentiation and 


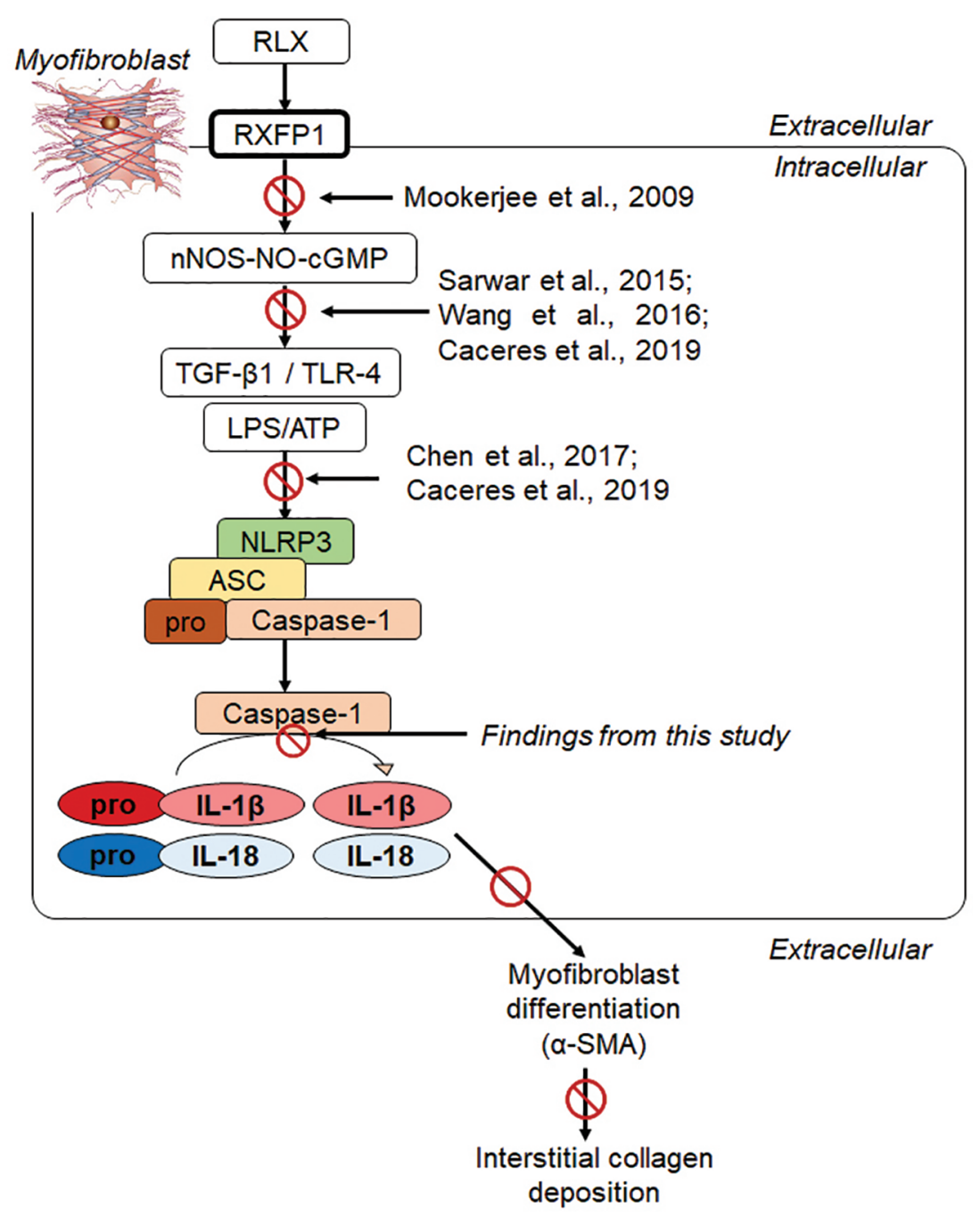

FIGURE 6 | Progressive studies that we and others have published, have led to our recent findings showing that RLX signaled through a RXFP1-nNOS-TLR-4dependent mechanism in human cardiac myofibroblasts to inhibit measures of myofibroblast NLRP3 inflammasome priming and activity, as well as myofibroblast differentiation and myofibroblast-induced collagen I deposition (Cáceres et al., 2019). We now show in this study that RLX can additionally modulate the myofibroblast NLRP3 inflammasome at the level of caspase-1 to mediate its anti-fibrotic actions; as the ability of RLX to inhibit IL-1 $\beta$, pro-IL-18, myofibroblast differentiation and collagen I deposition was specifically abrogated by siRNA-induced knock-down of caspase-1, but not of NLRP3 or ASC. Our collective findings suggest that RLX can target the TGF- $\beta 1$-TLR- 4 axis (upstream of the NLRP3 inflammasome) to inhibit NLRP3, ASC and pro-caspase- 1 ; and also target caspase-1 activity (within the NLRP3 inflammasome) to inhibit the pro-fibrotic contributions of IL-1 $\beta$ and IL-18 that is produced by the NLRP3 inflammasome, on myofibroblast differentiation ( $\alpha$-SMA expression) and collagen I deposition.

collagen I deposition was specifically abrogated by siRNA-induced knock-down of caspase-1, but not of NLRP3 or ASC. Collectively, our findings suggest firstly that RLX (acting through RXFP1) can target the TGF- $\beta 1$-TLR- 4 axis (upstream of the NLRP3 inflammasome) to inhibit NLRP3 and ASC; and secondly, through disruption of the TGF- $\beta 1$-TLR-4 axis and pro-caspase 1 (within the inflammasome), RLX can down-regulate caspase-1 activity to dampen the pro-fibrotic contributions of IL-1 $1 \beta$ and IL18 , that is produced by the myofibroblast NLRP3 inflammasome, on myofibroblast differentiation and collagen-I deposition (Figure 6). Our added findings that RLX could disrupt TGF- $\beta 1$ - induced myofibroblast differentiation and collagen-I deposition, in the absence of L+A-induced NLRP3 inflammasome activity, additionally suggests that RLX does not exclusively regulate the myofibroblast NLRP3 inflammasome to mediate its anti-fibrotic actions. Consistent with this, studies have shown that RLX can also suppress the upregulation of several chemokines and cytokines independently of IL-1 $\beta$ and/or IL-18 regulation (reviewed in Bathgate et al., 2013; Samuel et al., 2017). Our findings show that RLX is able to disrupt the TGF- $\beta 1-I L-1 \beta$ and TGF- $\beta 1$-IL-18 axes through the NLRP3 inflammasome on myofibroblasts. 
Furthermore, our findings shown in Supplementary Table 1 suggest that pro-caspase-1 and caspase-1 activity can be regulated independently of NLRP3 and/or ASC. As T-alone (without $\mathrm{L}+\mathrm{A}$ ) was found to stimulate pro-caspase-1 protein expression after $72 \mathrm{~h}$ (Supplementary Table 1), in the absence of NLRP3 or ASC in HDMFs, this provides evidence to suggest that pro-caspase-1 can be regulated independently of NLRP3 and ASC, and independently of NLRP3 inflammasome induction. This notion is in line with previous studies which demonstrated that caspase- 1 activity can be regulated upstream of the NLRP3 inflammasome, at the level of TLR- 4 and NF- $\kappa B$ activation (Kahlenberg et al., 2005; Franchi et al., 2009). On the other hand, as $\mathrm{T}+\mathrm{L}+\mathrm{A}$ stimulation promoted NLRP3-dependent caspase- 1 and IL-1 $\beta$ activity in HDMFs, this confirmed the NLRP3-dependent induction of caspase-1 and IL-1 $\beta$ activity, consistent with previous studies (Martinon et al., 2002; Sutterwala et al., 2006; Bauernfeind et al., 2009; Cáceres et al., 2019). Therefore, it is possible that caspase-1 can be regulated via NLRP3-dependent and -independent mechanisms. Hence, further work is required to determine how RLX specifically targets caspase- 1 activity on myofibroblasts.

Nevertheless, our findings overlap but are distinct from a previous study, which demonstrated that RLX could attenuate caspase-1 activity in an in vivo model of ischemia/reperfusion injury via an eNOS-dependent mechanism (Valle Raleigh et al., 2017), in which the direct involvement of the NLRP3 inflammasome was not provided (Valle Raleigh et al., 2017). As NO has been found to inhibit caspase-1, IL-1 $\beta$, and IL-18 release from macrophages (Collino et al., 2013), it was suggested that the ability of RLX to promote eNOS-induced NO correlated with the RLX-induced attenuation of NLRP3 inflammasome-induced caspase- 1 that was measured in the murine model studied (Valle Raleigh et al., 2017). However, several lines of evidence have indicated RLX to signal via a nNOS-dependent mechanism in myofibroblasts. Rat renal myofibroblasts (Mookerjee et al., 2009; Chow et al., 2012) and human cardiac myofibroblasts (Sarwar et al., 2015) express nNOS and iNOS but lack eNOS, with RLX shown to specifically up-regulate nNOS expression in these myofibroblasts (Mookerjee et al., 2009; Chow et al., 2012; Sarwar et al., 2015). Furthermore, pharmacological blockade of nNOS activity in rat renal myofibroblasts has been shown to inhibit the anti-fibrotic effects of RLX on TGF- $\beta 1$ signal transduction and myofibroblast differentiation (Mookerjee et al., 2009). Therefore, it would appear that RLX signals through a nNOS-dependent mechanism in myofibroblasts to inhibit TLR-4-NLRP3 inflammasome-caspase$1-\mathrm{IL}-1 \beta / \mathrm{IL}-18$ activity. On the other hand, RLX may regulate the NLRP3 inflammasome in other cells such as macrophages through an eNOS-NO- (Sogawa et al., 2018) or iNOS-NO- (Mao et al., 2013) dependent mechanism. Irrespective of how NO is produced by various cells, both our findings and those of Valle Raleigh (Valle Raleigh et al., 2017) and colleagues suggest that the RLX-induced promotion of $\mathrm{NO}$ can target caspase-1 release and activity within the NLRP3 inflammasome in multiple cell types, and its subsequent ability to activate IL-1 $\beta$ and IL- 18 .

The expression and activity of the NLRP3 inflammasome from different cell types (including various innate immune cells and myofibroblasts) is likely to be varied during wound healinginduced inflammation, tissue remodeling and eventually fibrogenesis (Yin et al., 2009). During the inflammatory phase of the wound healing response, the macrophage NLRP3 inflammasome is highly expressed and plays a fundamental role in secreting IL-1 $\beta$ and IL-18, which along with the TGF- $\beta 1$ that is secreted by macrophages, contribute to the activation of resident ECM-producing fibroblasts (Wynn and Barron, 2010). Although not expressed as highly as their macrophage counterparts, myofibroblast NLRP3 inflammasome components, such as NLRP3 and ASC, have been detected during the early stages of wound healing in response to injury (Mezzaroma et al., 2011; Gan et al., 2018). However, the IL-1 $\beta$ that is secreted by the myofibroblast NLRP3 inflammasome has been demonstrated to promote the effects of TGF- $\beta 1$ on human fibroblast proliferation and ECM synthesis, eventually contributing to adverse tissue remodeling and fibrosis (Vesey et al., 2002). Similarly, the epithelial cell NLRP3 inflammasome has been found to contribute to epithelialmesenchymal-transition (EMT) during wound healing-associated inflammation in response to tissue injury, which over time, contributes to fibrogenesis via an interaction with TGF- $\beta 1$ (Liu, 2004; Wang et al., 2013). Hence, further work is required to determine whether RLX universally targets NLRP3 inflammasome activity, and indeed the activity of other inflammasomes that contribute to fibrosis progression, or has temporal effects in suppressing over-activated inflammasome activity in a celldependent manner, to mediate its anti-fibrotic effects.

The findings from this study have provided further insight into the molecular mechanisms by which RLX mediates its antifibrotic actions on TGF- $\beta 1$ signal transduction and its interaction with the myofibroblast NLRP3 inflammasome. It is now clear that RLX can act at multiple levels to disrupt the profibrotic influence of TGF- $\beta 1$, through its ability to promote nNOS-induced NO and cGMP in myofibroblasts. RLX can disrupt TGF- $\beta 1$ signal transduction at the level of Smad2 and/ or Smad3 (Heeg et al., 2005; Mookerjee et al., 2009; Sassoli et al., 2013; Samuel et al., 2014; Wang et al., 2016; Kocan et al., 2017). It can also ameliorate the interaction between TGF- $\beta 1$ and TLR-4 (up-stream of the NLRP3 inflammasome) (Cáceres et al., 2019) and caspase- 1 activity (at the level of the NLRP3 inflammasome) to suppress IL- $1 \beta$ and IL-18 activity, as well as the interaction between TGF- $\beta 1$ and secreted IL-1 $\beta$ (down-stream of the NLRP3 inflammasome) to mediate its anti-fibrotic actions (Figure 6). In summary, these findings highlight the therapeutic relevance of targeting the myofibroblast NLRP3 inflammasome as a means of treating the fibrosis that is a key cause of tissue dysfunction and failure. In this regard, natural (Molteni et al., 2018) and pharmacological inhibitors (Matsunaga et al., 2011) of TLR-4, NLRP3 (Coll et al., 2015), caspase-1 (Kudelova et al., 2015), or IL-1 $\beta$ (Kudelova et al., 2015; Emmi et al., 2018) activity may limit the contribution of the NLRP3 inflammasome to fibrosis progression, while avoiding the redundancy and side-effects associated with TGF- $\beta 1$ blockade alone. However, future studies aimed at combining these drugs with RLX may lead to more efficacious blockade of fibrosis through suppression of the TGF- $\beta 1$-TLR-4-NLRP3 inflammasome axis. 


\section{DATA AVAILABILITY STATEMENT}

All datasets generated for this study are included in the article/ Supplementary Material.

\section{AUTHOR CONTRIBUTIONS}

AP and CS conceived, designed and supervised the study. AP, AY, and TG performed all the experiments. AP, AY, TG, and CS analyzed the data. AP, AY, TG, and CS contributed to writing of the manuscript.

\section{REFERENCES}

Aoki, H., Ohnishi, H., Hama, K., Ishijima, T., Satoh, Y., Hanatsuka, K., et al. (2006). Autocrine loop between TGF-betal and IL-1beta through Smad3- and ERK-dependent pathways in rat pancreatic stellate cells. Am. J. Physiol. Cell. Physiol. 290, C1100-C1108. doi: 10.1152/ajpcell.00465.2005

Artlett, C. M., and Thacker, J. D. (2015). Molecular activation of the NLRP3 Inflammasome in fibrosis: common threads linking divergent fibrogenic diseases. Antioxid. Redox Signal. 22, 1162-1175. doi: 10.1089/ars.2014.6148

Bathgate, R. A., Halls, M. L., Van Der Westhuizen, E. T., Callander, G. E., Kocan, M., and Summers, R. J. (2013). Relaxin family peptides and their receptors. Physiol. Rev. 93, 405-480. doi: 10.1152/physrev.00001.2012

Bauernfeind, F. G., Horvath, G., Stutz, A., Alnemri, E. S., MacDonald, K., Speert, D., et al. (2009). Cutting edge: NF- $\kappa B$ activating pattern recognition and cytokine receptors license NLRP3 inflammasome activation by regulating NLRP3 expression. J. Immunol. 183, 787-791. doi: 10.4049/jimmunol.0901363

Beiert, T., Tiyerili, V., Knappe, V., Effelsberg, V., Linhart, M., Stockigt, F., et al. (2017). Relaxin reduces susceptibility to post-infarct atrial fibrillation in mice due to anti-fibrotic and anti-inflammatory properties. Biochem. Biophys. Res. Commun. 490, 643-649. doi: 10.1016/j.bbrc.2017.06.091

Bonniaud, P., Margetts, P. J., Ask, K., Flanders, K., Gauldie, J., and Kolb, M. (2005). TGF-beta and Smad3 signaling link inflammation to chronic fibrogenesis. J. Immunol. 175, 5390-5395. doi: 10.4049/jimmunol.175.8.5390

Boza, P., Ayala, P., Vivar, R., Humeres, C., Caceres, F. T., Munoz, C., et al. (2016). Expression and function of toll-like receptor 4 and inflammasomes in cardiac fibroblasts and myofibroblasts: IL-1beta synthesis, secretion, and degradation. Mol. Immunol. 74, 96-105. doi: 10.1016/j.molimm.2016.05.001

Cáceres, F. T., Gaspari, T. A., Samuel, C. S., and Pinar, A. A. (2019). Serelaxin inhibits the pro-fibrotic TGF-beta1/IL-1beta axis by targeting TLR- 4 and the NLRP3 inflammasome on myofibroblasts. FASEB J. 33, 14717-14733. doi: 10.1096/fj.201901079RR

Chen, L., Sha, M. L., Li, D., Zhu, Y. P., Wang, X. J., Jiang, C. Y., et al. (2017). Relaxin abrogates renal interstitial fibrosis by regulating macrophage polarization via inhibition of Toll-like receptor 4 signaling. Oncotarget 8, 21044-21053. doi: 10.18632/oncotarget.15483

Chow, B. S., Chew, E. G., Zhao, C., Bathgate, R. A., Hewitson, T. D., and Samuel, C. S. (2012). Relaxin signals through a RXFP1-pERK-nNOS-NO-cGMP-dependent pathway to up-regulate matrix metalloproteinases: the additional involvement of iNOS. PloS One 7, e42714. doi: 10.1371/journal.pone.0042714

Coll, R. C., Robertson, A. A., Chae, J. J., Higgins, S. C., Munoz-Planillo, R., Inserra, M. C., et al. (2015). A small-molecule inhibitor of the NLRP3 inflammasome for the treatment of inflammatory diseases. Nat. Med. 21, 248-255. doi: $10.1038 / \mathrm{nm} .3806$

Collino, M., Rogazzoi, M., Pini, A., Benetti, E., Rosa, A. C., Chiazza, F., et al. (2013). Acute treatment with relaxin protects the kidney against ischaemia/ reperfusion injury. J. Cell. Mol. Med. 17, 1494-1505. doi: 10.1111/ jcmm. 12120

Du, X. J., Bathgate, R. A., Samuel, C. S., Dart, A. M., and Summers, R. J. (2010). Cardiovascular effects of relaxin: from basic science to clinical therapy. Nat. Rev. Cardiol. 7, 48-58. doi: 10.1038/nrcardio.2009.198

Emmi, G., Urban, M. L., Imazio, M., Gattorno, M., Maestroni, S., Lopalco, G., et al. (2018). Use of Interleukin-1 blockers in pericardial and cardiovascular diseases. Curr. Cardiol. Rep. 20, 61. doi: 10.1007/s11886-018-1007-6

\section{FUNDING}

This research was supported in part by a Monash Biomedicine Discovery Institute Fellowship to CS.

\section{SUPPLEMENTARY MATERIAL}

The Supplementary Material for this article can be found online at: https://www.frontiersin.org/articles/10.3389/fphar.2020.01201/ full\#supplementary-material

Franchi, L., Eigenbrod, T., Muñoz-Planillo, R., and Nuñez, G. (2009). The inflammasome: a caspase-1-activation platform that regulates immune responses and disease pathogenesis. Nat. Immunol. 10, 241-247. doi: 10.1038/ ni.1703

Frangogiannis, N. G. (2019). Cardiac fibrosis: Cell biological mechanisms, molecular pathways and therapeutic opportunities. Mol. Aspects Med. 65, 70-99. doi: 10.1016/j.mam.2018.07.001

Gan, W., Ren, J., Li, T., Lv, S., Li, C., Liu, Z., et al. (2018). The SGK1 inhibitor EMD638683, prevents Angiotensin II-induced cardiac inflammation and fibrosis by blocking NLRP3 inflammasome activation. Biochim. Biophys. Acta Mol. Basis. Dis. 1864, 1-10. doi: 10.1016/j.bbadis.2017.10.001

Gasse, P., Mary, C., Guenon, I., Noulin, N., Charron, S., Schnyder-Candrian, S., et al. (2007). IL-1R1/MyD88 signaling and the inflammasome are essential in pulmonary inflammation and fibrosis in mice. J. Clin. Invest. 117, 3786-3799. doi: $10.1172 / \mathrm{JCI} 32285$

Heeg, M. H., Koziolek, M. J., Vasko, R., Schaefer, L., Sharma, K., Muller, G. A., et al. (2005). The antifibrotic effects of relaxin in human renal fibroblasts are mediated in part by inhibition of the Smad2 pathway. Kidney Int. 68, 96-109. doi: 10.1111/j.1523-1755.2005.00384.x

Hossain, M. A., Rosengren, K. J., Samuel, C. S., Shabanpoor, F., Chan, L. J., Bathgate, R. A. D., et al. (2011). The minimal active structure of human relaxin-2. J. Biol. Chem. 286, 37555-37565. doi: 10.1074/jbc.M111.282194

Humeres, C., and Frangogiannis, N. G. (2019). Fibroblasts in the infarcted, remodeling, and failing heart. JACC Basic Transl. Sci. 4, 449-467. doi: 10.1016/j.jacbts.2019.02.006

Kahlenberg, J. M., Lundberg, K. C., Kertesy, S. B., Qu, Y., and Dubyak, G. R. (2005). Potentiation of caspase- 1 activation by the $\mathrm{P} 2 \mathrm{X} 7$ receptor is dependent on TLR signals and requires NF-אB-driven protein synthesis. J. Immunol. 175, 7611-7622. doi: 10.4049/jimmunol.175.11.7611

Kocan, M., Sarwar, M., Ang, S. Y., Xiao, J., Marugan, J. J., Hossain, M. A., et al. (2017). ML290 is a biased allosteric agonist at the relaxin receptor RXFP1. Sci. Rep. 7, 2968-2982. doi: 10.1038/s41598-017-02916-5

Kolb, M., Margetts, P. J., Anthony, D. C., Pitossi, F., and Gauldie, J. (2001). Transient expression of IL-1beta induces acute lung injury and chronic repair leading to pulmonary fibrosis. J. Clin. Invest. 107, 1529-1536. doi: 10.1172/ JCI12568

Kudelova, J., Fleischmannova, J., Adamova, E., and Matalova, E. (2015). Pharmacological caspase inhibitors: research towards therapeutic perspectives. J. Physiol. Pharmacol. 66, 473-482. doi: 10.1038/nm.3806

Li, L., Zhao, Q., and Kong, W. (2018). Extracellular matrix remodeling and cardiac fibrosis. Matrix Biol. 68-69, 490-506. doi: 10.1016/j.matbio.2018.01.013

Liu, Y. (2004). Epithelial to mesenchymal transition in renal fibrogenesis: pathologic significance, molecular mechanism, and therapeutic intervention. J. Am. Soc Nephrol. 15, 1-12. doi: 10.1097/01.asn.0000106015.29070.e7

Mangan, M. S. J., Olhava, E. J., Roush, W. R., Seidel, H. M., Glick, G. D., and Latz, E. (2018). Targeting the NLRP3 inflammasome in inflammatory diseases. Nat. Rev. Drug Discovery 17, 588-606. doi: 10.1038/nrd.2018.97

Mao, K., Chen, S., Chen, M., Ma, Y., Wang, Y., Huang, B., et al. (2013). Nitric oxide suppresses NLRP3 inflammasome activation and protects against LPSinduced septic shock. Cell Res. 23, 201-212. doi: 10.1371/journal.pone.0203823

Martinon, F., Burns, K., and Tschopp, J. (2002). The inflammasome: a molecular platform triggering activation of inflammatory caspases and processing of proIL-1ß. Mol. Cell. 10, 417-426. doi: 10.1016/s1097-2765(02)00599-3 
Matsunaga, N., Tsuchimori, N., Matsumoto, T., and Ii, M. (2011). TAK-242 (resatorvid), a small-molecule inhibitor of toll-like receptor (TLR)-4 signaling, binds selectively to TLR- 4 and interferes with interactions between TLR- 4 and its adaptor molecules. Mol. Pharmacol. 79, 34-41. doi: 10.1124/mol.110.068064

Mezzaroma, E., Toldo, S., Farkas, D., Seropian, I. M., Van Tassell, B. W., Salloum, F. N., et al. (2011). The inflammasome promotes adverse cardiac remodeling following acute myocardial infarction in the mouse. Proc. Natl. Acad. Sci. U.S.A. 108, 19725-19730. doi: 10.1073/pnas.1108586108

Molteni, M., Bosi, A., and Rossetti, C. (2018). Natural products with toll-like receptor 4 antagonist activity. Int. J. Inflam. 2018, 2859135-2859144. doi: $10.1155 / 2018 / 2859135$

Mookerjee, I., Hewitson, T. D., Halls, M. L., Summers, R. J., Mathai, M. L., Bathgate, R. A., et al. (2009). Relaxin inhibits renal myofibroblast differentiation via RXFP1, the nitric oxide pathway, and Smad2. FASEB J. 23, 1219-1229. doi: 10.1096/fj.08-120857

Nistri, S., Bigazzi, M., and Bani, D. (2007). Relaxin as a cardiovascular hormone: physiology, pathophysiology and therapeutic promises. Cardiovasc. Hematol. Agents Med. Chem. 5, 101-108. doi: 10.2174/187152507780363179

Rodriguez-Alcazar, J. F., Ataide, M. A., Engels, G., Schmitt-Mabmunyo, C., Garbi, N., Kastenmuller, W., et al. (2019). Charcot-Leyden crystals activate the NLRP3 inflammasome and cause IL-1beta inflammation in human macrophages. J. Immunol. 202, 550-558. doi: 10.4049/jimmunol.1800107

Samuel, C. S., Unemori, E. N., Mookerjee, I., Bathgate, R. A., Layfield, S. L., Mak, J., et al. (2004). Relaxin modulates cardiac fibroblast proliferation, differentiation and collagen production and reverses cardiac fibrosis in vivo. Endocrinology 145, 4125-4133. doi: 10.1210/en.2004-0209

Samuel, C. S., Bodaragama, H., Chew, J. Y., Widdop, R. E., Royce, S. G., and Hewitson, T. D. (2014). Serelaxin is a more efficacious antifibrotic than enalapril in an experimental model of heart disease. Hypertension 64, 315322. doi: 10.1161/HYPERTENSIONAHA.114.03594

Samuel, C. S., Royce, S. G., Hewitson, T. D., Denton, K. M., Cooney, T. E., and Bennett, R. G. (2017). Anti-fibrotic actions of relaxin. Br. J. Pharmacol. 174, 962-976. doi: 10.1111/bph.13529

Sarwar, M., Samuel, C. S., Bathgate, R. A., Stewart, D. R., and Summers, R. J. (2015). Serelaxin-mediated signal transduction in human vascular cells: bellshaped concentration-response curves reflect differential coupling to $G$ proteins. Br. J. Pharmacol. 172, 1005-1019. doi: 10.1111/bph.12964

Sassoli, C., Chellini, F., Pini, A., Tani, A., Nistri, S., Nosi, D., et al. (2013). Relaxin prevents cardiac fibroblast-myofibroblast transition via notch-1-mediated inhibition of TGF-beta/Smad3 signaling. PloS One 8, e63896-e63906. doi: 10.1371/journal.pone.0063896

Schroer, A. K., and Merryman, W. D. (2015). Mechanobiology of myofibroblast adhesion in fibrotic cardiac disease. J. Cell Sci. 128, 1865-1875. doi: 10.1242/ jcs.162891

Sogawa, Y., Nagasu, H., Itano, S., Kidokoro, K., Taniguchi, S., Takahashi, M., et al. (2018). The eNOS-NO pathway attenuates kidney dysfunction via suppression of inflammasome activation in aldosterone-induced renal injury model mice. PloS One 13, e0203823-e0203840. doi: 10.1371/journal.pone.0203823

Sutterwala, F. S., Ogura, Y., Szczepanik, M., Lara-Tejero, M., Lichtenberger, G. S., Grant, E. P., et al. (2006). Critical role for NALP3/CIAS1/Cryopyrin in innate and adaptive immunity through its regulation of caspase-1. Immunity $24,317-$ 327. doi: 10.1016/j.immuni.2006.02.004

Unemori, E. N., and Amento, E. P. (1990). Relaxin modulates synthesis and secretion of procollagenase and collagen by human dermal fibroblasts. J. Biol. Chem. 265, 10681-10685.

Unemori, E. N., Pickford, L. B., Salles, A. L., Piercy, C. E., Grove, B. H., Erikson, M. E., et al. (1996). Relaxin induces an extracellular matrix-degrading phenotype in human lung fibroblasts in vitro and inhibits lung fibrosis in a murine model in vivo. J. Clin. Invest. 98, 2739-2745. doi: 10.1172/JCI119099

Valle Raleigh, J., Mauro, A. G., Devarakonda, T., Marchetti, C., He, J., Kim, E., et al. (2017). Reperfusion therapy with recombinant human relaxin-2 (Serelaxin) attenuates myocardial infarct size and NLRP3 inflammasome following ischemia/reperfusion injury via eNOS-dependent mechanism. Cardiovasc. Res. 113, 609-619. doi: 10.1093/cvr/cvw246

Vesey, D. A., Cheung, C., Cuttle, L., Endre, Z., Gobe, G., and Johnson, D. W. (2002). Interleukin-1beta stimulates human renal fibroblast proliferation and matrix protein production by means of a transforming growth factor-beta-dependent mechanism. J. Lab. Clin. Med. 140, 342-350. doi: 10.1067/mlc.2002.128468

Wang, W., Wang, X., Chun, J., Vilaysane, A., Clark, S., French, G., et al. (2013). Inflammasome-independent NLRP3 augments TGF-beta signaling in kidney epithelium. J. Immunol. 190, 1239-1249. doi: 10.4049/jimmunol.1201959

Wang, C., Kemp-Harper, B. K., Kocan, M., Ang, S. Y., Hewitson, T. D., and Samuel, C. S. (2016). The anti-fibrotic actions of relaxin are mediated through a NO-sGC-cGMP-dependent pathway in renal myofibroblasts in vitro and enhanced by the NO donor, diethylamine NONOate. Front. Pharmacol. 7, 91. doi: 10.3389/fphar.2016.00091

Wynn, T. A., and Barron, L. (2010). Macrophages: master regulators of inflammation and fibrosis. Semin. Liver Dis. 30, 245-257. doi: 10.1055/s-0030-1255354

Wynn, T. A., and Ramalingam, T. R. (2012). Mechanisms of fibrosis: therapeutic translation for fibrotic disease. Nat. Med. 18, 1028-1040. doi: 10.1038/nm.2807

Wynn, T. A. (2008). Cellular and molecular mechanisms of fibrosis. J. Pathol. 214, 199-210. doi: 10.1002/path.2277

Yin, Y., Yan, Y., Jiang, X., Mai, J., Chen, N. C., Wang, H., et al. (2009). Inflammasomes are differentially expressed in cardiovascular and other tissues. Int. J. Immunopathol. Pharmacol. 22, 311-322. doi: 10.1177/ 039463200902200208

Zhou, W., Chen, C., Chen, Z., Liu, L., Jiang, J., Wu, Z., et al. (2018). NLRP3: a novel mediator in cardiovascular disease. J. Immunol. Res. 2018, 5702103-5702111. doi: $10.1155 / 2018 / 5702103$

Conflict of Interest: The authors declare that the research was conducted in the absence of any commercial or financial relationships that could be construed as a potential conflict of interest.

Copyright (C) 2020 Pinar, Yuferov, Gaspari and Samuel. This is an open-access article distributed under the terms of the Creative Commons Attribution License (CC BY). The use, distribution or reproduction in other forums is permitted, provided the original author(s) and the copyright owner(s) are credited and that the original publication in this journal is cited, in accordance with accepted academic practice. No use, distribution or reproduction is permitted which does not comply with these terms. 